\title{
Spare guanylyl cyclase NO receptors ensure high NO sensitivity in the vascular system
}

\author{
Evanthia Mergia, Andreas Friebe, Oliver Dangel, Michael Russwurm, and Doris Koesling
}

Institut für Pharmakologie und Toxikologie, Ruhr-Universität Bochum, Bochum, Germany.

\begin{abstract}
In the vascular system, the receptor for the signaling molecule NO, guanylyl cyclase (GC), mediates smooth muscle relaxation and inhibition of platelet aggregation by increasing intracellular cyclic GMP (cGMP) concentration. The heterodimeric GC exists in 2 isoforms $\left(\alpha_{1}-\mathrm{GC}, \alpha_{2}-\mathrm{GC}\right)$ with indistinguishable regulatory properties. Here, we used mice deficient in either $\alpha_{1}$ - or $\alpha_{2}$-GC to dissect their biological functions. In platelets, $\alpha_{1}$-GC, the only isoform present, was responsible for NO-induced inhibition of aggregation. In aortic tissue, $\alpha_{1}$-GC, as the major isoform (94\%), mediated vasodilation. Unexpectedly, $\alpha_{2}-\mathrm{GC}$, representing only $6 \%$ of the total GC content in WT, also completely relaxed $\alpha_{1}$-deficient vessels albeit higher NO concentrations were needed. The functional impact of the low cGMP levels produced by $\alpha_{2}$-GC in vivo was underlined by pronounced blood pressure increases upon NO synthase inhibition. As a fractional amount of GC was sufficient to mediate vasorelaxation at higher NO concentrations, we conclude that the majority of NO-sensitive GC is not required for cGMP-forming activity but as NO receptor reserve to increase sensitivity toward the labile messenger NO in vivo.
\end{abstract}

\section{Introduction}

The NO/cyclic GMP (NO/cGMP) signaling cascade is involved in the regulation of a variety of physiological responses and has an established importance in the vascular system (1-3). Disorders in the NO/cGMP signaling cascade have been implicated in the development of endothelial dysfunction, a state that often precedes cardiovascular diseases. NO continuously formed in the endothelium by eNOS in response to shear stress causes smooth muscle relaxation and inhibition of platelet aggregation. Both effects are mediated by the receptor for NO, the NO-sensitive guanylyl cyclase (GC) present in smooth muscle cells and in platelets. NO-sensitive GC catalyzes the formation of cGMP, and the subsequent cGMP increase leads to activation of the cGMP-dependent protein kinase I (cGKI) and further transduction of the NO signal $(4,5)$. Cyclic GMP is also formed by membrane GC receptors (GC-A) that are stimulated by peptide hormones, e.g., atrial natriuretic peptide (ANP) (6). The cGMP signal is terminated by the action of the cyclic nucleotide-hydrolyzing phosphodiesterases (PDEs), with PDE1, PDE3, and PDE5 being of importance in the vascular system (7).

Due to its key role in NO/cGMP signaling, NO-sensitive GC is an important drug target (8), and NO-releasing compounds used for more than a century are still among the most effective drugs in the treatment of coronary heart disease. NO-sensitive GC exists in 2 isoforms containing either an $\alpha_{1}$ or $\alpha_{2}$ subunit dimerized to the $\beta_{1}$ subunit $\left(\alpha_{1}-\mathrm{GC}\right.$ and $\left.\alpha_{2}-\mathrm{GC}\right)$ with indistinguishable enzymatic properties (9). The specific role and importance of the isoforms are unknown. Yet a special subcellular localization for $\alpha_{2}-\mathrm{GC}$ is conceivable, as the $\alpha_{2}$ subunit contains a PDZ-recognition domain and its interaction with the postsynaptic adapter protein PSD95 has been demonstrated (10). The question remains whether the

Nonstandard abbreviations used: ANP, atrial natriuretic peptide; cGKI, cGMPdependent protein kinase I; cGMP, cyclic GMP; DEA-NO, 2-( $N, N$-diethylamino)diazenolate-2-oxide; GC, guanylyl cyclase; GSNO, S-nitrosoglutathione; ODQ, $1 H$-[1,2,4] oxadiazol[4,3-a]quinoxalin-1-one; PCPT, p-chlorophenylthio; PDE, phosphodiesterase; RIA, radioimmunoassay.

Conflict of interest: The authors have declared that no conflict of interest exists. Citation for this article: J. Clin. Invest. 116:1731-1737 (2006). doi:10.1172/JCI27657.
2 isoforms of NO-sensitive GC serve diverse biological functions or whether 1 isoform can substitute for the other. To address this question, we generated mice in which either the $\alpha_{1}$ or the $\alpha_{2}$ subunit was deleted $\left(\alpha_{1}-\mathrm{KO}, \alpha_{2}-\mathrm{KO}\right)$.

Here we report on the vascular properties of the knockout mice. Whereas in platelets only the $\alpha_{1}$-GC was present and responsible for NO-induced inhibition of platelet aggregation, in aortic tissue, both isoforms occurred and elicited vasorelaxation. Unexpectedly, the $\alpha_{2}-\mathrm{GC}$, representing only $6 \%$ of the total GC content in aortic tissue, was sufficient to induce the full biological response at higher NO concentrations. These data demonstrate that the majority of guanylyl cyclase NO receptors function as spare receptors to increase NO sensitivity and point to bioavailability of $\mathrm{NO}$ as the critical parameter within NO/cGMP signaling.

\section{Results}

The $2 \alpha$ subunits of GC are encoded by different genes. To generate the $\mathrm{KO}$ mice, we used Cre/loxP-mediated recombination to delete exon 4 of either the $\alpha_{1}$ or $\alpha_{2}$ subunit, respectively (Figure 1). Homozygous $\alpha_{1}$-KO and $\alpha_{2}$-KO mice were born at normal Mendelian ratio and did not show apparent alterations or a reduced life expectancy. To confirm the loss of the $\alpha_{1}$ or $\alpha_{2}$ subunit, we performed Western blot analysis using subunit-specific antibodies. As can be seen in Figure 2A, the proteins were completely absent in lung and brain of the KO mice. WT-like amounts of the other, nondeleted $\alpha$ subunit were determined in the respective $\mathrm{KO}$ animals, indicating that deletion of $1 \alpha$ subunit is not compensated by upregulation of the other. Interestingly, the loss of the $\alpha$ subunit was accompanied by a decrease in the $\beta$ subunit content, indicating that the $\beta$ subunit was not stable without its dimerizing partner. The observed reduction of the $\beta$ subunit revealed similar expression of $\alpha_{1}$ and $\alpha_{2}$ isoforms in brain as well as the dominance of $\alpha_{1}$-GC in lung.

The NO-dependent activity in $\alpha_{1}$ - and $\alpha_{2}$-KO mice represents the amount of the respective nondeleted GC isoform in a tissue. Thus, we measured NO-stimulated cGMP formation in lung and brain of $\mathrm{KO}$ and WT animals (Figure 2B). NO-dependent GC activities 

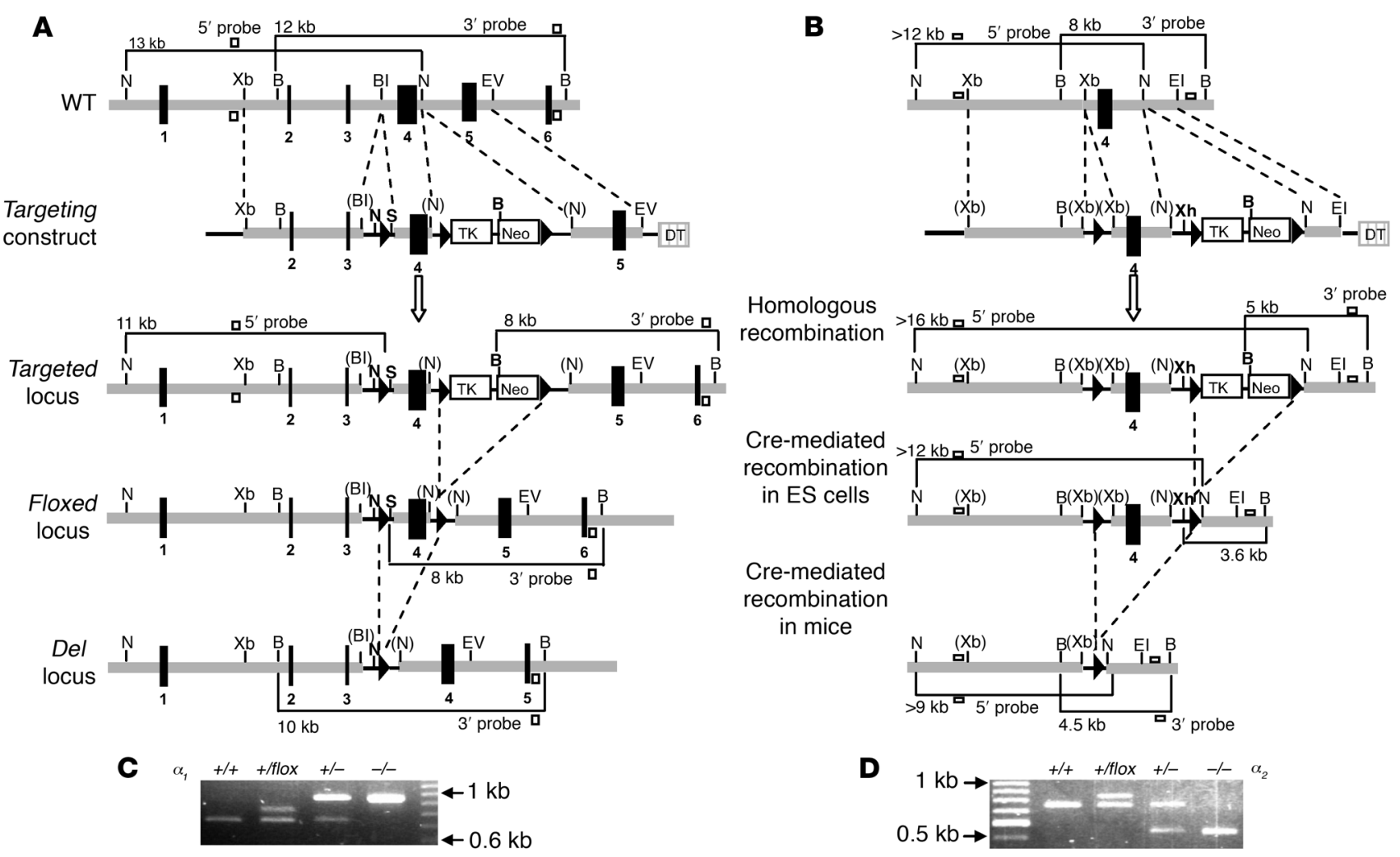

Figure 1

Gene inactivation of $\alpha$ subunits of NO-sensitive GC. Targeting strategies for $\alpha_{1}(\mathbf{A})$ or $\alpha_{2}$ (B) subunit of NO-sensitive GC. Arrowheads, loxP sites; Neo, neomycin resistance; TK, thymidine kinase; DT, diphtheria toxin gene; WT, wild-type loci of the $\alpha$ subunits; filled rectangles, exons, numbered from the first coding exon. B, BamHI; BI, Bglll; El, EcoRl; EV, EcoRV; N, Nhel; S, Spel; Xb, Xbal, Xh, Xhol. Brackets indicate destroyed restriction sites; newly created sites are in bold. Genotyping of $\alpha_{1}(\mathbf{C})$ or the $\alpha_{2}$ (D) mouse line was performed with PCR analysis as described in Methods.

in brain amounted to $1.4 \pm 0.1 \mathrm{nmol} / \mathrm{mg} / \mathrm{min}$ in WT and were reduced by about $50 \%$ in $\alpha_{1}$ - and $\alpha_{2}$-deficient animals $(0.5 \pm 0.1$ and $0.9 \pm 0.2 \mathrm{nmol} / \mathrm{mg} / \mathrm{min}$, respectively). In lung, WT-like activity was measured in $\alpha_{2}-\mathrm{KO}(4.5 \pm 0.7 \mathrm{nmol} / \mathrm{mg} / \mathrm{min})$ whereas GC activity in $\alpha_{1}-\mathrm{KO}$ was greatly reduced (7\% of WT). These findings are in agreement with the Western blot results and confirm $\alpha_{1}$-GC as the major isoform in lung and similar expression of both isoforms in brain.

In platelets, $\mathrm{NO}$ inhibits aggregation via the stimulation of $\mathrm{NO}-$ sensitive GC and the subsequent increase in cGMP. Platelets from $\alpha_{2}$-KO mice showed WT-like behavior; i.e., collagen-induced aggregation was completely inhibited by NO (Figure 3A). In contrast, $\alpha_{1}$-deficient platelets did not respond to $\mathrm{NO}$ at all. Accordingly, in Western blots, only the $\alpha_{1}$ subunit was detected in WT platelets (Figure 3B) whereas the $\alpha_{2}$ subunit was undetectable (data not shown), indicating that $\alpha_{2}$-GC does not exist in this cell type. These results demonstrate that inhibition of platelet aggregation by $\mathrm{NO}$ is solely mediated by $\alpha_{1}-\mathrm{GC}$.

To assess the physiological impact of the GC isoforms on vascular tone, we studied $\mathrm{NO}$-induced relaxation of aortic rings of $\alpha_{1}$ and $\alpha_{2}$-deficient mice. Concentration-response curves for the NO donor S-nitrosoglutathione (GSNO) revealed WT-like behavior of $\alpha_{2}$-deficient aortic rings (half-maximal effective concentration $\left.\left[\mathrm{EC}_{50}\right] \cong 0.4 \mu \mathrm{M}\right)$, underlining the predominant role of $\alpha_{1}$-GC in vasorelaxation (Figure 4A). Unexpectedly, $\alpha_{1}$-deficient rings were completely relaxed by GSNO as well. However, the concentration-response curve for $\mathrm{NO}$ was shifted to the right with a 5-fold higher $\mathrm{EC}_{50}(2 \mu \mathrm{M})$. These findings demonstrate an unpredicted functional role of $\alpha_{2}$-GC in vascular relaxation. To ensure that the retained $\mathrm{NO}$-induced relaxation in $\alpha_{1}$-deficient rings was caused by $\mathrm{NO}$-sensitive $\mathrm{GC}$ and not by other $\mathrm{NO}$ effector molecules, we used the inhibitor of NO-sensitive GC, $1 H$-[1,2,4] oxadiazol[4,3-a] quinoxalin-1-one (ODQ) (11). First, ODQ effects were established in the WT rings to confirm complete inhibition of NO-sensitive GC under the applied conditions. As can be seen in Figure $4 \mathrm{~B}$, in the presence of ODQ, NO-induced relaxation of WT rings was fully abolished. The responsiveness of the ODQ-treated rings was confirmed by the subsequent relaxation with ANP. Moreover, ODQ also inhibited the endothelium-dependent relaxation induced by carbachol, demonstrating its inhibitory potential toward endogenously produced NO (Figure 4D). When ODQ was applied to $\alpha_{1}$-deficient rings, the vascular response to $\mathrm{NO}$ was completely abolished, confirming $\alpha_{2}-\mathrm{GC}$ as the NO receptor mediating relaxation in $\alpha_{1}-\mathrm{KO}$ (Figure 4B). The results are further supported by the inhibition of carbachol-induced relaxation in the $\alpha_{1}$-deficient rings by ODQ as well (Figure 4D). By measuring NOstimulated GC activity in $\alpha_{1}$-deficient aortic tissue (Figure $4 \mathrm{C}$ ), $\alpha_{2}$-GC was determined to represent only $6 \%$ of the total GC content $\left(\alpha_{1}-\mathrm{KO}, 0.24 \pm 0.15 \mathrm{nmol} / \mathrm{mg} / \mathrm{min}\right.$ versus WT, $3.8 \pm 0.8 \mathrm{nmol} /$ $\mathrm{mg} / \mathrm{min})$. Taken together, the retained relaxation in the $\alpha_{1}$-defi- 

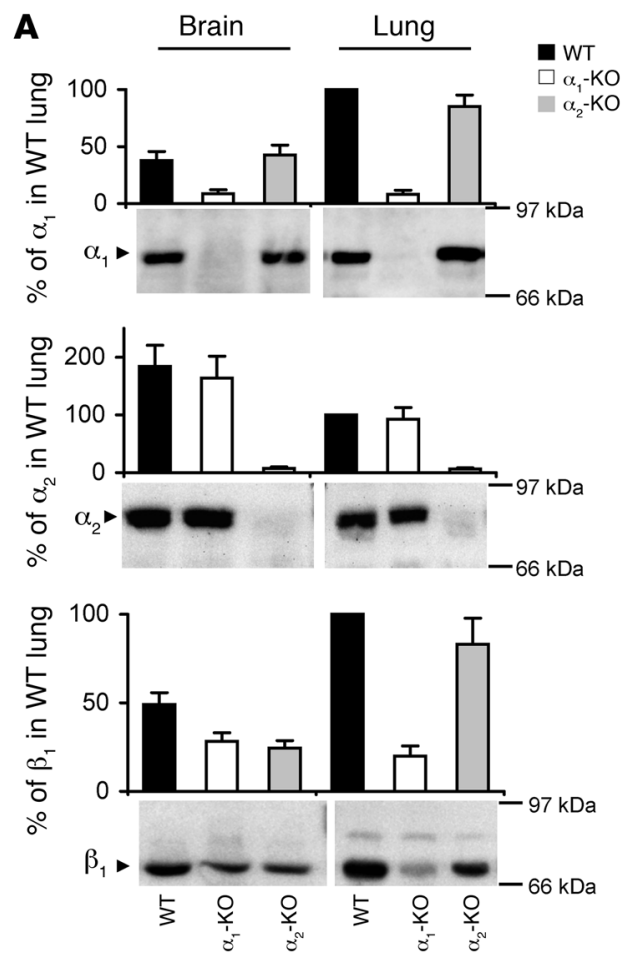

B

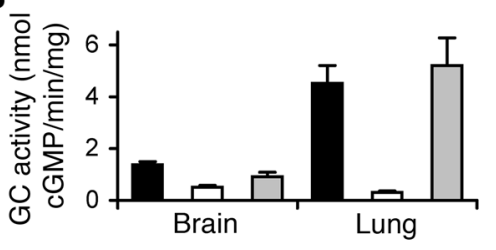

cient rings shows that the loss of the majority of the cGMP-forming NO receptor (94\%) does not impair the biological response to but decreases the potency of NO. To study the physiological cGMP response, we induced endothelium-dependent relaxation using carbachol (Figure 4D). Here, a carbachol concentration $(30 \mu \mathrm{M})$ that evoked an almost complete relaxation of WT and $\alpha_{2}$-deficient aortic rings (approximately $90 \%$ ) induced $50 \%$ relaxation in the $\alpha_{1}$-deficient rings. Obviously, the cGMP increase caused by endogenously produced $\mathrm{NO}$ is able to induce significant relaxation in the $\alpha_{1}$-deficient rings; thus, small increases in cGMP exert a profound effect on vascular tone.

In an attempt to judge the cGMP increases in aorta, we determined the cGMP content in aortic rings with and without exogenous NO as well as in the presence of the GC inhibitor ODQ (Figure 5). In WT and $\alpha_{2}$-deficient aortas, steady state levels of cGMP $(\sim 12 \mathrm{pmol} / \mathrm{mg})$ were comparable and were significantly reduced by ODQ $(\sim 6 \mathrm{pmol} / \mathrm{mg})$, demonstrating the impact of endogenously produced NO. The addition of NO yielded compa-

\section{Figure 3}

Analysis of $\alpha$-deficient platelets. (A) Aggregation of $\alpha$-deficient platelets was induced with collagen $(4 \mu \mathrm{g} / \mu \mathrm{l})$ in the absence or presence of DEA-NO $(100 \mu \mathrm{M})$. Shown are aggregometric traces. (B) To identify the $\mathrm{GC}$ isoform expressed in platelets, Western blot analysis of platelet homogenates from $\mathrm{WT}, \alpha_{1}-\mathrm{KO}$, and $\alpha_{2}-\mathrm{KO}$ mice was performed.

\section{Figure 2}

Analysis of GC isoform content in $\alpha-\mathrm{KO}$ mice. (A) GC subunit content in brain and lung homogenates analyzed with antibodies against the different subunits $\left(\alpha_{1}, \alpha_{2}\right.$, and $\left.\beta_{1}\right)$ and quantified with respect to the subunit amount in WT lung (100\%; $n=4$ of each genotype). (B) Cyclic GMP-forming activity in brain and lung homogenates in the presence of DEA-NO $(100 \mu \mathrm{M})$ was determined as outlined in detail in Methods ( $n=5$ of each genotype).

rable cGMP increases in WT and $\alpha_{2}$-deficient rings. In contrast, cGMP levels in the $\alpha_{1}$-deficient aortic rings did not differ in the absence or presence of ODQ $(\sim 6 \mathrm{pmol} / \mathrm{mg})$ and did not significantly increase upon addition of NO. The results show that the cGMP formed by the residual $\alpha_{2}$-GC is not detectable and strongly suggest that local cGMP increases are responsible for the induction of the biological response.

To detect a putative increased responsiveness toward cGMP in the rings with the low GC content, we induced relaxation with the stimulator of the membrane-bound GC-A, ANP, and the direct activator of the cGKI, 8-(p-chlorophenylthio)-cGMP (8-pCPT-cGMP) (Figure 6). An increased sensitivity toward both substances was observed in the $\alpha_{1}$-deficient rings, indicating an as yet unknown regulatory potential within the cGMP signaling cascade. We then asked whether the observed increase in ANP sensitivity was supported by a reduction of the cGMP-degrading PDEs. We evaluated the different PDE isoforms present in aortas (PDE1, PDE3, and PDE5) by measuring PDE activities under various experimental conditions (different substrate concentrations, presence of milrinone, sildenafil, EGTA, $\mathrm{Ca}^{2+} /$ calmodulin; Supplemental Table 1; supplemental material available online with this article; doi:10.1172/JCI27657DS1). However, we did not find any significant reduction of cGMP-degrading activities in the $\alpha_{1}$-deficient aortas.

The importance of NO/cGMP signaling for the maintenance of blood pressure is well established. To monitor the impact of the GC isoforms, systolic blood pressure was assessed by tail-cuff measurements. The systolic values obtained in the $\alpha_{2}-\mathrm{KO}$ animals did not differ significantly from those in WT, as shown in Figure 7A, whereas blood pressure in $\alpha_{1}$-deficient mice was moderately elevated (mean $111 \pm 2 \mathrm{mmHg}$ versus $104 \pm 2 \mathrm{mmHg}$ ). These results indicate that $\alpha_{2}-\mathrm{GC}$ was not sufficient to completely substitute for the loss
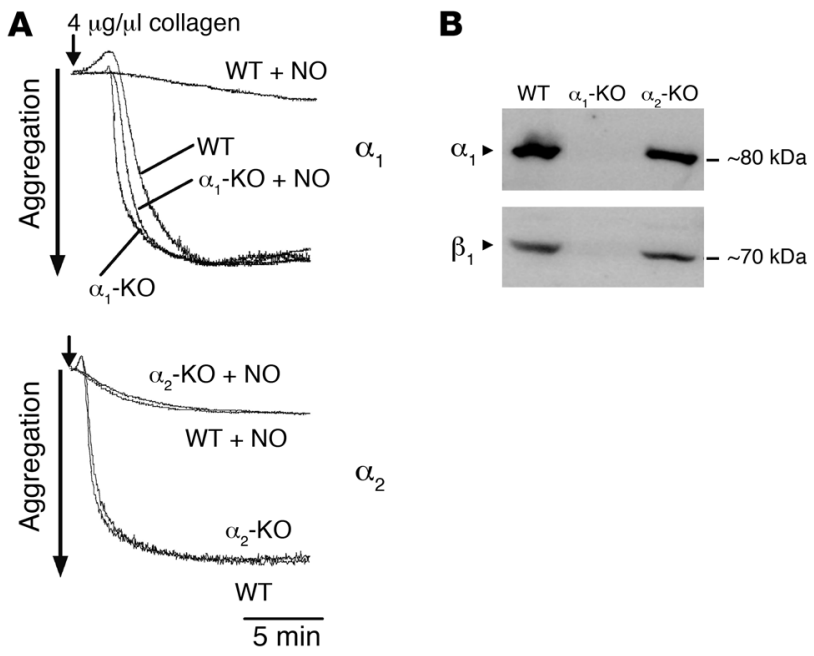
A

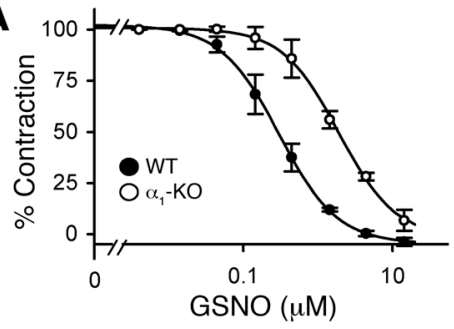

B
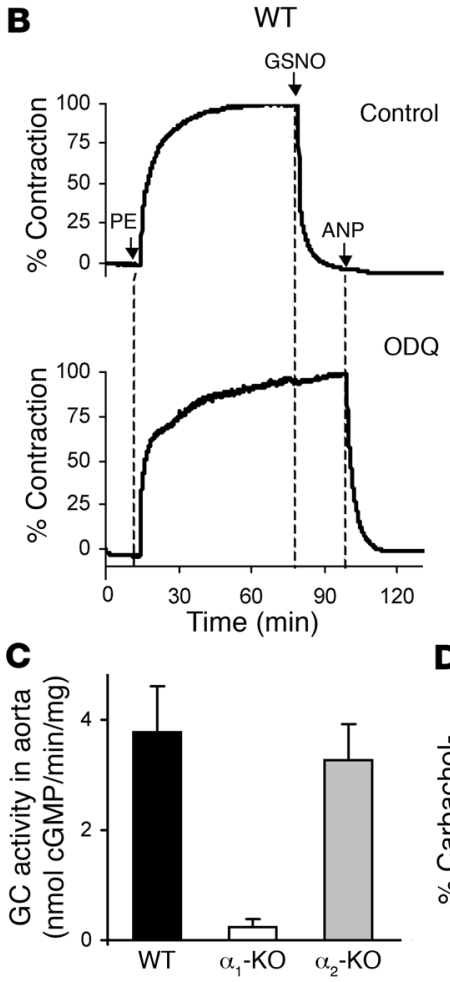

D

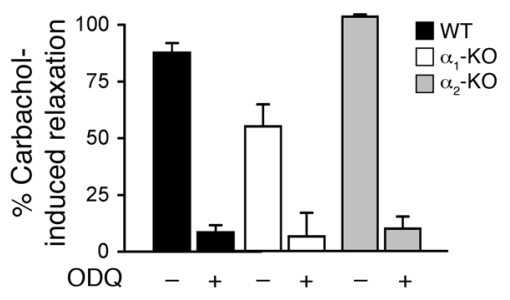

\section{Figure 4}

Vasorelaxing properties of $\alpha$-deficient aortic rings. Functional responses of aortic rings were determined as described in Methods. (A) Cumulative concentration-response curves of GSNO-induced relaxation of $\alpha_{1}$ - and $\alpha_{2}$-deficient rings ( $n=3$ in each group). (B) GSNO-induced relaxation of WT and $\alpha_{1}$-deficient rings in the presence of the inhibitor of NO-sensitive GC, ODQ. Shown are the original recordings using $10 \mu \mathrm{M}$ GSNO and $20 \mu \mathrm{M}$ ODQ. ANP (10 nM) was added to confirm integrity of the rings. (C) NO-stimulated GC activities (100 $\mu \mathrm{M}$ DEA-NO) determined in aortic homogenates ( $n=5$ per genotype). (D) Carbachol-induced relaxation of WT and $\alpha$-deficient aortic rings in the absence or presence of ODQ ( $n=3-8$ per genotype). Experiments used $30 \mu \mathrm{M}$ carbachol and $20 \mu \mathrm{M}$ ODQ.

Platelets contain high levels of NO-sensitive GC, and the rise in the intraplatelet cGMP concentration induced by endothelium-derived NO causes inhibition of aggregation $(14,15)$. The complete lack of NO-induced inhibition of aggregation in $\alpha_{1}$-deficient platelets revealed that the antiaggregatory effect is solely mediated by the $\alpha_{1}$-GC. In aortic smooth muscle cells, in contrast, $\alpha_{2}$-GC was present and sufficient to mediate NO- and carbachol-induced relaxation despite its low content (6\%). Moreover, the complete inhibition of both $\mathrm{NO}$ - and carbachol-induced relaxation by the inhibitor of NO-sensitive GC, ODQ, demonstrates that NO signals entirely via both GC isoforms, excluding other NO targets at least in blood vessel relaxation. The results demonstrate that the $\alpha_{2}$-GC is able to bring about the same physiological response as the major $\alpha_{1}-\mathrm{GC}$ in smooth muscle cells. The role of the $\alpha_{2}$-GC in regulation of smooth muscle tone is further underlined by blood pressure analysis after inhibition of endogenous NO production.

$\mathrm{NO} / \mathrm{cGMP}$ signaling is regarded as an important determinant for the regulation of blood pressure (16). As expected from the greatly reduced GC content, $\alpha_{1}-\mathrm{KO}$ animals showed a discrete elevation of systolic blood presof the major $\alpha_{1}$-GC. To confirm the functional role of $\alpha_{2}-\mathrm{GC}$ in the $\alpha_{1}$-KOs, mice were treated with the NO synthase inhibitor L-NAME (Figure 6B). L-NAME treatment led to an increase in blood pressure in the $\alpha_{1}$-KO mice (21\%), demonstrating that NO-induced cGMP synthesis, although being greatly reduced in these mice, retains its importance for regulation of smooth muscle tone in vivo.

\section{Discussion}

We explored the roles of the GC isoforms in the vascular system by using $2 \mathrm{KO}$ lines in which the respective isoform-specific $\alpha$ subunit was deleted. Due to the enzyme's heterodimeric structure, the removal of either of the $\alpha$ subunits led to a loss of the dimerizing $\beta_{1}$ subunit on the protein level. Importantly, the loss of one of the GC isoforms was not compensated by an upregulation of the remaining one. Hence, the cGMP-forming activity in the KO animals stands for the residual nondeleted GC isoform $\left(\alpha_{1}-\mathrm{GC}\right.$ or $\left.\alpha_{2}-\mathrm{GC}\right)$. Comparatively high levels of $\alpha_{2}$-GC were identified in brain, where the amount of this isoform equaled the amount of $\alpha_{1}$-GC. $\alpha_{1}-\mathrm{GC}$ was the only isoform in platelets and the dominant isoform (>90\%) in lung and aorta. As both KO lines showed a normal life expectancy, the amounts of cGMP formed by either one of the GC isoforms was apparently sufficient to prevent the high mortality reported for the KO mice of cGKI (12) or its substrate IRAG (13). sure (7\%). As blood pressure elevation in $\mathrm{KO}$ mice lacking the endothelial NO synthase (17) or the cGKI (12) was more pronounced $(-15 \%)$, we conclude that $\alpha_{2}-\mathrm{GC}$, the remaining GC isoform in the $\alpha_{1}$-deficient animals, prevents further elevation of blood pressure. L-NAME treatment of $\alpha_{1}$-KO mice led to an increase in blood pressure thereby demonstrating the relevance

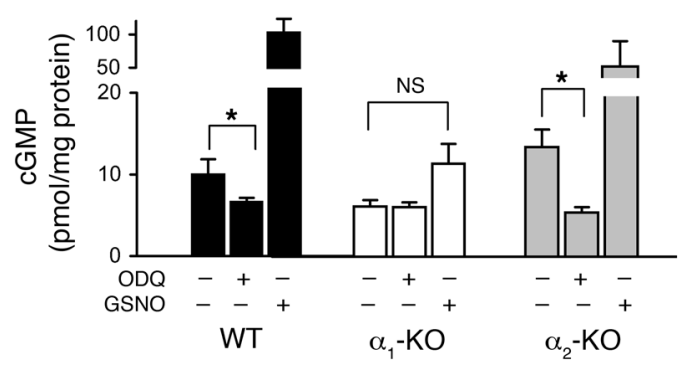

Figure 5

Cyclic GMP levels determined in intact aortic rings of WT, $\alpha_{1-}^{-}$, and $\alpha_{2}$-deficient mice. Cyclic GMP content was determined in rings incubated without any addition or in the presence of ODQ (20 $\mu \mathrm{M}, 15 \mathrm{~min})$ or GSNO (100 $\mu \mathrm{M}, 5 \mathrm{~min})\left(n=3-6\right.$ per genotype). ${ }^{*} P<0.05$, differences considered significant. 

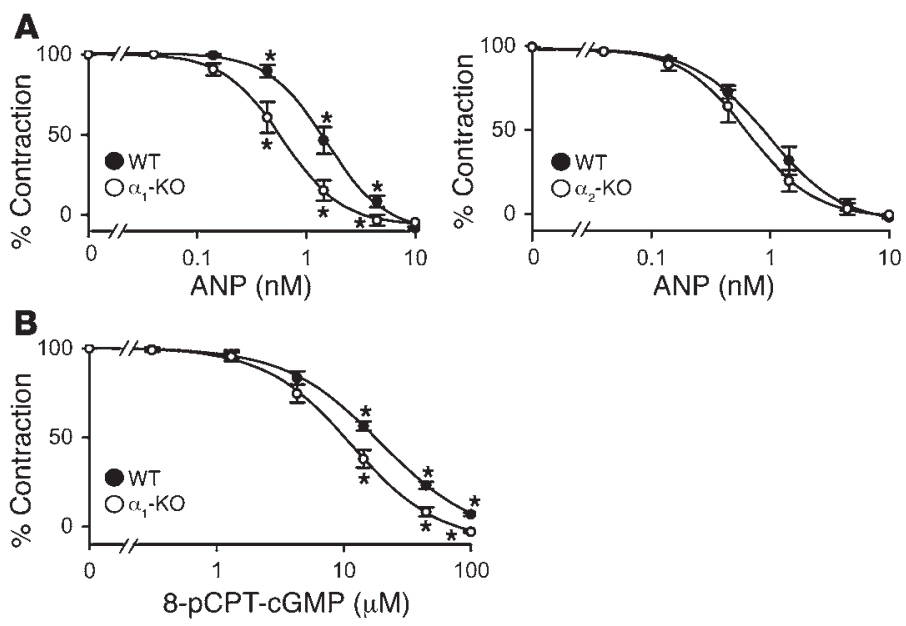

of the fractional amount of cGMP formed by the $\alpha_{2}$-GC in the regulation of blood pressure.

Yet the aortic rings with low $\alpha_{2}$-GC content showed an increased responsiveness toward ANP and the cGMP analogue 8-pCPTcGMP, indicating an as yet unknown regulatory potential on the level of cGKI or beyond. An augmented vascular reactivity toward cGMP has not been observed in endothelial NO synthase-deficient mice $(18,19)$. A reduced sensitivity toward cGMP-induced relaxation has been reported to occur during heart failure, and downregulation of the myosin light chain phosphatase, one of the postulated targets of the cGKI, has been suggested as the underlying mechanism (20). The mechanism(s) responsible for the observed change in vascular reactivity in the $\alpha_{1}$-deficient mice are not known but are of great pathophysiological interest as factors adjusting vascular resistance.

As the increased sensitivity toward ANP could be assisted by a reduction of the cGMP-hydrolyzing PDEs, we extensively compared PDE activities in WT and $\alpha_{1}$-deficient aortas. Despite the reports on downregulation of PDE5 in endothelial NO synthasedeficient KO mice (21) and upregulation of PDE1 in the presence of high NO (22), we did not observe a significant reduction of PDE activity and conclude that the deletion of the majority of NO-sensitive GC is not paralleled by a reduced expression of either one of the aortic PDEs.

By characterization of $\alpha_{1}$ - and $\alpha_{2}$-deficient animals, we demonstrate for the first time to our knowledge that $\alpha_{2}-\mathrm{GC}$, in addition to the major $\alpha_{1}$-GC, mediates smooth muscle relaxation, indicating that the $2 \mathrm{GC}$ isoforms can serve the same function in certain cells. Moreover, our study reveals that very low amounts of cGMP are sufficient for a full biological response, as $94 \%$ of cGMP-forming

\section{Figure 7}

Systolic blood pressure in $\alpha$-deficient mice. (A) Systolic blood pressure as measured in conscious mice by tail-cuff plethysmography as described in Methods. Means (indicated by horizontal lines) of 111 and $104 \mathrm{mmHg}$ were determined for the $\alpha_{1}-\mathrm{KO}$ mice and their WT siblings, respectively; for the $\alpha_{2}-\mathrm{KO}$ mice and the respective WT littermates, means of 111 and $110 \mathrm{mmHg}$ were determined. The $95 \%$ confidence interval of the difference between $\alpha_{1}-\mathrm{KO}$ and WT mice is $1-15 \mathrm{mmHg}$. (B) Increase of blood pressure induced by L-NAME treatment of $\alpha_{1}$-KO mice ( $n=5$ per genotype). ${ }^{\star} P<0.05$, differences considered significant.

\section{Figure 6}

Increased vascular reactivity of the $\alpha_{1}$-deficient aortic rings. (A) Cumulative concentration-response curves of ANP-induced relaxation of $\alpha_{1}$ - and $\alpha_{2}$-deficient aortic rings ( $n=3$ per genotype). (B) Cumulative concentration-response curves of 8-pCPT-cGMPinduced relaxation in the $\alpha_{1}$-deficient aortic rings ( $n=4$ per genotype). ${ }^{\star} P<0.05$, differences considered significant.

activity can be eliminated without abolishing vasorelaxation. The increased NO concentration required for this response is explained by the drastic reduction of the NO receptor. Apparently, the majority of NO-sensitive GC is not required because of its cGMP-forming capacity but as a NO receptor reserve to increase the potency of NO. These findings fit well with the concept of spare receptors, in which additional receptors exist to increase the sensitivity toward the ligand and ensure that the biological response is not limited on the level of the receptor. Accordingly, available NO is the critical parameter within $\mathrm{NO} / \mathrm{cGMP}$ signaling, and spare $\mathrm{NO}$ receptors are required to compete with other $\mathrm{NO}$ scavengers in vivo. Our results agree with the finding that endothelial dysfunction is based on reduced NO bioavailability (23). Pharmacologically, NO sensitizers such as YC-1 (24) and related substances (25) that increase the sensitivity of NO-sensitive GC toward NO may offer a therapeutic approach to compensate for an NO deficiency under such conditions.

\section{Methods}

Generation of $\alpha_{1}$ or $\alpha_{2}$ KO mice. Murine genomic DNAs of the $\alpha_{1}$ and $\alpha_{2}$ subunit were derived from bacterial artificial chromosome (BAC) clones (Incyte Genomics) containing genomic DNA of ES cells of 129/SvJ mice. The targeting vectors (Figure 1, A and B) were constructed such that exon 4
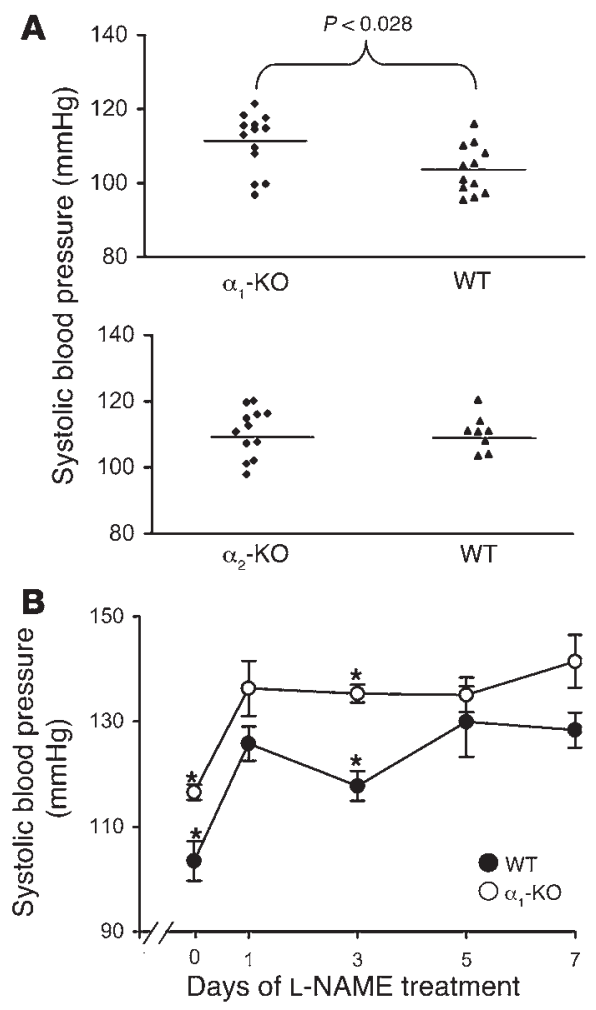
of the $\alpha_{1}$ or $\alpha_{2}$ subunit (numbering from the first coding exon) was flanked by a loxP-Tk/Neo-loxP cassette and a single loxP site using a triple loxP vector system (26). Chimeras were bred with $\mathrm{C} 57 \mathrm{BL} / 6$ mice, and the resulting heterozygotes $\left(\alpha_{1}^{+f f l o x}\right.$ and $\left.\alpha_{2}^{+f f l o x}\right)$ were crossed with the general deleter mouse EIIa-Cre (27), resulting in the deletion of the floxed exon $4\left(\mathrm{\alpha}_{1}^{+/-}\right.$and $\mathrm{\alpha}_{2}^{+--}$). To remove the Cre recombinase gene, the heterozygous KO mice were crossed with WT mice (C57BL/6). Finally, the generated heterozygotes were intercrossed to yield homozygous mice $\left(\alpha_{1}^{-/-}\right.$and $\left.\alpha_{2}^{-/-}\right)$.

Genotyping of the animals was performed by PCR analysis of tail DNA (Figure 1, C and D). The $\alpha_{1}$-WT (879 bp) and $\alpha_{1}$-floxed (920 bp) fragments were amplified by the primer pair $\mathrm{F}_{1} \mathrm{U}_{1}$ : ATGACAAATGAGCAGACG and $\mathrm{F}_{1} \mathrm{~L}_{1}$ : TCCCGAGATGAAGTAGTTAGTA, and $\alpha_{1}$-del (997 bp) fragment by the primer pair loxP- $\alpha_{1} \mathrm{U}_{1}$ : TGTAGAAGAGGGGATAGAAAGACC and $\mathrm{F}_{1} \mathrm{~L}_{1}$. The $\alpha_{2}$-WT (760 bp) and $\alpha_{2}$-floxed (800 bp) fragments were amplified by the primer pair $\mathrm{F}_{2} \mathrm{U}_{1}$ : TTTGAAATTACTTGGAGATAGA and $\mathrm{F}_{2} \mathrm{~L}_{2}$ : AACTATGTAATTATCAACTGG. An $\alpha_{2}$-del (513 bp) fragment was detected with the primer pair $P \alpha_{2} \mathrm{U}_{5}$ : AGGTGGGGCTGTCTCTGAA and $\mathrm{F}_{2} \mathrm{~L}_{1}$ : GGGGGCCCTGACATTTGA. Studies were performed with adult mice (2-4 months old) of either sex with a hybrid background (129/SvJ $\times$ C57BL/6; F2-F3 generation). In each experiment, either litter- or age-matched mice of the same sex were used wherever feasible.

Preparations of murine tissues. After decapitation, organs (brain, lung, aorta free of surrounding tissue) were removed and homogenized immediately in buffer $(50 \mathrm{mM}$ triethanolamine/ $\mathrm{HCl}, 50 \mathrm{mM} \mathrm{NaCl}, 1 \mathrm{mM}$ EDTA, $2 \mathrm{mM}$ DTT, $0.2 \mathrm{mM}$ benzamidine, $0.5 \mathrm{mM}$ phenylmethylsulfonyl fluoride (PMSF), and $1 \mu \mathrm{M}$ pepstatin $\mathrm{A} ; \mathrm{pH} 7.4,4^{\circ} \mathrm{C}$ ). After centrifugation $\left(800 \mathrm{~g}, 5 \mathrm{~min}, 4^{\circ} \mathrm{C}\right)$, homogenates were subjected to further experiments. The protein concentration was determined in triplicate and repeated 3 times (Bradford; Bio-Rad Laboratories).

Western blot analysis. Separation of proteins (homogenates $20 \mu \mathrm{g} / \mathrm{lane}$ or $5 \times 10^{7}$ platelets/lane), blotting, detection, and quantification of the signals were performed as described (28). The signals were standardized relative to the amount of the respective protein in WT lung. Western blot analysis of each animal was carried out in duplicate and repeated twice $(n=4$ of each genotype). Anti- $\alpha_{2}$ antibodies were raised and purified as described (10); polyclonal antibodies against the $\alpha_{1}$ and $\beta_{1}$ subunits were raised by immunizing rabbits with the respective $\mathrm{N}$ terminal fragments as glutathione-Stransferase-fusion proteins $\left(\alpha_{1}\right.$, aa $1-259 ; \beta_{1}$, aa $\left.1-370\right)$ and subsequently purified using the appropriate fragments covalently immobilized on glutathione-Sepharose (GSH-Sepharose; Amersham Biosciences).

Determination of NO-stimulated GC activity. NO-stimulated GC activity was measured for 10 minutes $\left(37^{\circ} \mathrm{C}\right)$ in $1-5 \mu$ l of homogenates (10-30 $\mu$ g protein) using $100 \mu \mathrm{M}$ 2-( $N, N$-diethylamino)-diazenolate-2-oxide (DEA-NO; Alexis Corp.) as described (29). The cGMP formed by lung and aortic homogenates was detected by radioimmunoassay (RIA). In this case, the incubation of homogenate was carried out as described above except that less homogenate (2-3 $\mu$ g protein) was applied, cGMP was omitted, and the amount of GTP was lowered to $0.25 \mathrm{mM}$. The reactions were terminated by removing an aliquot $(10 \mu \mathrm{l})$ into ice cold RIA buffer ( $90 \mu \mathrm{l}: 100 \mathrm{mM} \mathrm{CH}_{3} \mathrm{COONa}$; $\mathrm{pH}$ 6.0). Preparation of tracer, acetylation of samples and standards, and incubation with antibody were performed as described (30). Assays were performed in triplicate with the subsequent detection of cGMP in RIAs in duplicate and were repeated twice. Shown in Figures $2 B$ and $4 C$ are the data (means \pm SEM) from 5 independent experiments.

Isolation and aggregation of murine platelets. All experiments were conducted in accordance with German legislation on protection of animals and approved by the Bezirksregierung Arnsberg, Arnsberg, Germany. Whole blood (approximately $800 \mu \mathrm{l}$ ) was collected from the orbital sinus of anesthetized mice in $200 \mu \mathrm{l}$ heparin solution $(50 \mathrm{U} / \mathrm{ml})$. After dilution $\left(1 \mathrm{ml} \mathrm{PBS}, \mathrm{Ca}^{2+}\right.$ - and $\mathrm{Mg}^{2+}$ free) and centrifugation $\left(90 \mathrm{~g}, 15 \mathrm{~min}, 20^{\circ} \mathrm{C}\right)$, platelet-rich plasma (PRP) was obtained. Optical aggregation of PRP $\left(2-3 \times 10^{7}\right.$ platelets $\left./ 200 \mu \mathrm{l}\right)$ was performed in a 4-channel aggregometer (Chrono-log Corp.) after addition of collagen (4 mg/ $\mu \mathrm{l}$; Chrono-log Corp.). When indicated, DEA-NO $(100 \mu \mathrm{M})$ was administered 3 minutes before addition of collagen.

Organ bath experiments with aortic rings. Thoracic aortic rings were mounted isometrically on fixed segment support pins in two 4-chamber myographs (Multi Myograph Model 610 M; Danish Myo Technology A/S) containing $5 \mathrm{ml}$ of Krebs-Henseleit buffer $(118 \mathrm{mM} \mathrm{NaCl}, 4.7 \mathrm{mM} \mathrm{KCl}$, $1.2 \mathrm{mM} \mathrm{MgSO}_{4}, 1.2 \mathrm{mM} \mathrm{KH}_{2} \mathrm{PO}_{4}, 25 \mathrm{mM} \mathrm{NaHCO}_{3}, 2.55 \mathrm{mM} \mathrm{CaCl}_{2}$, and $7.5 \mathrm{mM}$ D-glucose) and gassed with $5 \% \mathrm{CO}_{2}$ in $\mathrm{O}_{2}$. Diclofenac $(3 \mu \mathrm{mol} / \mathrm{l})$ and L-NAME (200 $\mu \mathrm{M}$; Sigma-Aldrich) were present unless indicated. Resting tension was set to $5 \mathrm{mN}$. After equilibration, rings were contracted (1 $\mu \mathrm{M}$ phenylephrine; Sigma-Aldrich), and the vasorelaxing effects of GSNO (Alexis Corp.), ANP (Sigma-Aldrich), and 8-pCPT-cGMP (Biolog Inc.) were assessed as cumulative concentration-response curves. ODQ (20 $\mu \mathrm{M}$; Alexis Corp.) was added 15 minutes before contraction. Vasorelaxation to carbachol (30 $\mu \mathrm{M}$; Sigma-Aldrich) was recorded in absence of L-NAME. Each experiment was performed in parallel with 4 aortic rings, each derived from a KO and a WT mouse, respectively.

Determination of CGMP content in intact aortic rings. Aortic rings (4 per aorta) were allowed to equilibrate for 30 minutes in temperate $\left(37^{\circ} \mathrm{C}\right)$, oxygenated (in $95 \% \mathrm{O}_{2}, 5 \% \mathrm{CO}_{2}$ ) Krebs-Henseleit buffer and thereafter were incubated with ODQ $(20 \mu \mathrm{M}, 15 \mathrm{~min})$ or GSNO $(100 \mu \mathrm{M}, 5 \mathrm{~min})$. The cGMP levels of equilibrated, untreated rings were taken as controls. To extract cGMP, rings were frozen in liquid nitrogen, homogenized in $70 \%(\mathrm{v} / \mathrm{v})$ ice cold ethanol using a glass/glass homogenizer, and then centrifuged $(14,000 \mathrm{~g}$, $15 \mathrm{~min}, 4^{\circ} \mathrm{C}$ ). Supernatants were dried at $95^{\circ} \mathrm{C}$, and the cGMP content was measured by RIA. In order to standardize the different samples, protein pellets were dissolved in $0.1 \mathrm{M} \mathrm{NaOH} / 0.1 \% \mathrm{SDS}$ and protein content determined using the bicinchoninic acid method (Uptima).

Measurement of $c$ GMP-bydrolyzing PDE activity. See Supplemental Methods. Measurement of blood pressure. Systolic blood pressure was measured in conscious mice by tail-cuff plethysmography (BP-98A; Softron Co.). Measurements were taken from a total of $13 \alpha_{1}$-KO mice ( 5 females and 8 males) compared with $12 \mathrm{WT}$ littermates ( 5 females and 7 males) and from $12 \alpha_{2}$-KO mice ( 4 females and 8 males) compared with 8 WT littermates ( 4 females and 4 males). For habituation, mice were trained daily (7 days). After the training period, 10 measurements per mouse were recorded daily for 5 days. L-NAME $(50 \mathrm{mg} / \mathrm{ml})$ was applied via drinking water (7 days), and blood pressure was measured 24 hours after the first application and then every 2 days.

Statistics. Data are expressed as mean $\pm \operatorname{SEM}(n=$ number of animals) and were compared by the paired Student's $t$ test or the independent 2-tailed $t$ test. Differences were considered significant at a $P$ value of less than 0.05 .

\section{Acknowledgments}

We thank Fred Eichhorst, Ursula Krabbe, and Medah Özcan for excellent technical assistance. Stephan Teglund (Karolinska Institutet, Department of Biosciences at Novum) is acknowledged for culturing the ES cells and generating the chimeras. This work is supported by a Deutsche Forschungsgemeinschaft grant.

Received for publication December 13, 2005, and accepted in revised form March 14, 2006.

Address correspondence to: Doris Koesling, Institut für Pharmakologie und Toxikologie, Medizinische Fakultät MA N1, Ruhr-Universität Bochum, 44780 Bochum, Germany. Phone: 49-234-322-6827; Fax: 49-234-321-4521; E-mail: doris.koesling@ ruhr-uni-bochum.de. 
1. Waldman, S.A., and Murad, F. 1987. Cyclic GMP synthesis and function. Pharmacol. Rev. 39:163-196.

2. Moncada, S., and Higgs, E.A. 1995. Molecular mechanisms and therapeutic strategies related to nitric oxide. FASEB J. 9:1319-1330.

3. Ignarro, L.J. 2002. Nitric oxide as a unique signaling molecule in the vascular system: a historical overview. J. Physiol. Pharmacol. 53:503-514.

4. Feil, R., Lohmann, S.M., de Jonge, H., Walter, U., and Hofmann, F. 2003. Cyclic GMP-dependent protein kinases and the cardiovascular system: insights from genetically modified mice. Circ. Res. 93:907-916

5. Munzel, T., et al. 2003. Physiology and pathophysiology of vascular signaling controlled by guanosine $3^{\prime}, 5^{\prime}$-cyclic monophosphate-dependent protein kinase. Circulation. 108:2172-2183.

6. Kuhn, M. 2003. Structure, regulation, and function of mammalian membrane guanylyl cyclase receptors, with a focus on guanylyl cyclase-A. Circ. Res. 93:700-709.

7. Rybalkin, S.D., Yan, C., Bornfeldt, K.E., and Beavo, J.A. 2003. Cyclic GMP phosphodiesterases and regulation of smooth muscle function. Circ. Res. 93:280-291.

8. Friebe, A., and Koesling, D. 2003. Regulation of nitric oxide-sensitive guanylyl cyclase. Circ. Res. 93:96-105.

9. Russwurm, M., and Koesling, D. 2002. Isoforms of NO-sensitive guanylyl cyclase. Mol. Cell. Biochem. 230:159-164.

10. Russwurm, M., Wittau, N., and Koesling, D. 2001. Guanylyl cyclase/PSD-95 interaction: targeting of the NO-sensitive $\alpha_{2} \beta_{1}$ guanylyl cyclase to synaptic membranes. J. Biol. Chem. 276:44647-44652.

11. Garthwaite, J., et al. 1995. Potent and selective inhi- bition of nitric oxide-sensitive guanylyl cyclase by 1H-[1,2,4]oxadiazolo[4,3-a]quinoxalin-1-one. $\mathrm{Mol}$. Pharmacol. 48:184-188.

12. Pfeifer, A., et al. 1998. Defective smooth muscle regulation in cGMP kinase I-deficient mice. EMBO J. 17:3045-3051.

13. Geiselhöringer, A., et al. 2004. IRAG is essential for relaxation of receptor-triggered smooth muscle contraction by cGMP kinase. EMBO J. 23:4222-4231.

14. Moncada, S., Radomski, M.W., and Palmer, R.M. 1988. Endothelium-derived relaxing factor. Identification as nitric oxide and role in the control of vascular tone and platelet function. Biochem. Pharmacol. 37:2495-2501.

15. Waldmann, R., and Walter, U. 1989. Cyclic nucleotide elevating vasodilators inhibit platelet aggregation at an early step of the activation cascade. Eur. J. Pharmacol. 159:317-320.

16. Vallance, P., Collier, J., and Moncada, S. 1989. Effects of endothelium-derived nitric oxide on peripheral arteriolar tone in man. Lancet. 2:997-1000.

17. Van Vliet, B.N., Chafe, L.L., and Montani, J.P. 2003. Characteristics of $24 \mathrm{~h}$ telemetered blood pressure in eNOS-knockout and C57Bl/6J control mice. J. Physiol. 549:313-325.

18. Hussain, M.B., Hobbs, A.J., and MacAllister, R.J. 1999. Autoregulation of nitric oxide-soluble guanylate cyclase-cyclic GMP signalling in mouse thoracic aorta. Br. J. Pharmacol. 128:1082-1088.

19. Brandes, R.P., et al. 2000. Increased nitrovasodilator sensitivity in endothelial nitric oxide synthase knockout mice: role of soluble guanylyl cyclase. Hypertension. 35:231-236.

20. Karim, S.M., et al. 2004. Vascular reactivity in heart failure: role of myosin light chain phosphatase. Circ. Res. 95:612-618.
21. Champion, H.C., Bivalacqua, T.J., Takimoto, E., Kass, D.A., and Burnett, A.L. 2005. Phosphodiesterase-5A dysregulation in penile erectile tissue is a mechanism of priapism. Proc. Natl. Acad. Sci. U. S. A. 102:1661-1666.

22. Kim, D., et al. 2001. Upregulation of phosphodiesterase $1 \mathrm{~A} 1$ expression is associated with the development of nitrate tolerance. Circulation. 104:2338-2343.

23. Münzel, T., Daiber, A., Ullrich, V., and Mülsch, A. 2005. Vascular consequences of endothelial nitric oxide synthase uncoupling for the activity and expression of the soluble guanylyl cyclase and the cGMP-dependent protein kinase. Arterioscler. Thromb. Vasc. Biol. 25:1551-1557.

24. Friebe, A., Schultz, G., and Koesling, D. 1996. Sensitizing soluble guanylyl cyclase to become a highly CO-sensitive enzyme. EMBO J. 15:6863-6868.

25. Stasch, J.P., et al. 2001. NO-independent regulatory site on soluble guanylate cyclase. Nature. 410:212-215.

26. Gainetdinov, R.R., et al. 2003. Dopaminergic supersensitivity in $G$ protein-coupled receptor kinase 6deficient mice. Neuron. 38:291-303.

27. Lakso, M., et al. 1996. Efficient in vivo manipulation of mouse genomic sequences at the zygote stage. Proc. Natl. Acad. Sci. U. S. A. 93:5860-5865.

28. Mergia, E., Russwurm, M., Zoidl, G., and Koesling, D. 2003. Major occurrence of the new $\alpha_{2} \beta_{1}$ isoform of NO-sensitive guanylyl cyclase in brain. Cell. Signal. 15:189-195.

29. Russwurm, M., and Koesling, D. 2005. Purification and characterization of NO-sensitive guanylyl cyclase. Methods Enzymol. 396:492-501.

30. Brooker, G., Harper, J.F., Terasaki, W.L., and Moylan, R.D. 1979. Radioimmunoassay of cyclic AMP and cyclic GMP. Adv. Cyclic Nucleotide Res. 10:1-33. 